\title{
O.S.P.
}

\section{L'évolution des intérêts des adolescents à la fin du collège : 1978-2004}

The evolution of interests of ninth grade students: 1978-2004

Yann Forner, Agnès Lasne-Cordonnier, Chantal-Marie Mercier et Hamida Salhi

\section{OpenEdition}

\section{Journals}

Édition électronique

URL : https://journals.openedition.org/osp/969

DOI : 10.4000/osp.969

ISSN : 2104-3795

Éditeur

Institut national d'étude du travail et d'orientation professionnelle (INETOP)

\section{Édition imprimée}

Date de publication : 15 septembre 2006

Pagination : 337-362

ISSN : 0249-6739

\section{Référence électronique}

Yann Forner, Agnès Lasne-Cordonnier, Chantal-Marie Mercier et Hamida Salhi, « L'évolution des intérêts des adolescents à la fin du collège : 1978-2004 ", L'orientation scolaire et professionnelle [En ligne], 35/3 | 2006, mis en ligne le 15 septembre 2009, consulté le 07 février 2023. URL : http:// journals.openedition.org/osp/969; DOI : https://doi.org/10.4000/osp.969

Ce document a été généré automatiquement le 7 février 2023

Tous droits réservés 


\section{L'évolution des intérêts des adolescents à la fin du collège : 1978-2004}

The evolution of interests of ninth grade students: 1978-2004

Yann Forner, Agnès Lasne-Cordonnier, Chantal-Marie Mercier et Hamida Salhi

\section{Introduction}

1 L'évolution des intérêts des adolescents est surtout connue pour des changements spécifiques comme l'actuelle « désaffection des étudiants pour les études scientifiques » (Ourisson, 2002). Dans de nombreux pays développés, les effectifs des étudiants en sciences ont tendance à diminuer de manière préoccupante pour le renouvellement des cadres scientifiques et techniques. Si les propositions d'action sont nombreuses, les propositions d'explication sont plus hésitantes: il apparait avant tout que les enseignements scientifiques sont jugés « peu attrayants » (voir aussi C.C.S.T.I.-Grenoble (2004) et Forner, Ferrer, Le Veler \& Thiéry (2006). Plus généralement, la question mérite d'être posée d'une évolution générale de ces "goûts ", c'est-à-dire aussi des intérêts scolaires et professionnels.

2 Ces intérêts sont traditionnellement définis comme « des tendances ou dispositions relativement stables ou durables (et dont le développement semble associé à celui de l'image de soi) orientées vers différents domaines d'objets ou, plus exactement, vers différents domaines d'activités et d'expériences vécues dans un milieu culturel donné. Ces tendances seraient également conditionnées par les pressions plus ou moins fortes définissant les rôles dévolus aux deux sexes» (Dupont, Gendre, Berthoud \& Descombes, 1979, p. 11).

3 Les études des intérêts, et notamment celles des intérêts inventoriés par des questionnaires, n'épuisent pas toute la diversité des préférences, mais ont généralement été organisées autour du devenir scolaire, universitaire et, in fine, 
professionnel des personnes. Il s'agissait initialement de prévoir le comportement spontané ultérieur en matière de choix professionnel et, ainsi, de fournir au conseiller les bases d'une recommandation, rigoureusement fondée, d'orientation vers une formation ou un emploi. Ces intentions peuvent encore demeurer mais il s'agit plutôt, maintenant, d'aider la personne à élaborer une représentation de soi comportant des descripteurs professionnels et, ainsi, de l'armer pour ses propres décisions d'orientation, actuelles ou futures. Cette centration sur les réalités professionnelles a mené à organiser le questionnement de ces épreuves, soit sur des professions, soit sur des activités quotidiennes évoquant ces professions.

4 Toutefois, la grande majorité des épreuves, et des théories qu'elles opérationnalisent, n'établissent pas de relations directes entre personnes et professions mais installent des construits intermédiaires. Ces construits, i.e. les caractéristiques mesurées, ont été d'abord surtout formulés en dimensions d'intérêts professionnels (Larcebeau, 1967, par exemple), ils le sont maintenant plutôt en types personnels et professionnels sous l'influence des propositions de Holland (1985). Même si les opérationnalisations de ces deux types de construits sont parfois confondues, leurs principes diffèrent fondamentalement : une approche dimensionnelle suppose une variation quantitative (quasiment continue) du construit, elle appelle une mesure par une échelle d'intervalle, elle vise à attribuer au sujet divers scores d'intérêt: littéraire, artistique ou scientifique, par exemple. Une approche typologique suppose une variation qualitative du construit, elle appelle une mesure par une échelle nominale, elle vise à qualifier - ou pas - le sujet de réaliste ou d'investigatif, par exemple.

5 Ces tendances ou dispositions vers différents domaines d'objets relèvent logiquement d'une psychologie de la motivation: l'étude des facteurs qui déclenchent l'activité, l'orientent, la maintiennent et la font cesser. Ainsi, selon Super (1964; voir aussi Tétreau, 2005), la notion fondamentale en matière de motivation serait celle de besoin, qui désignerait la forme la plus simple de rapport au monde, surtout lorsqu'il s'agit de besoins fondamentaux (de type déficience ou carence), mais aussi quand il s'agit de besoins psychologiques élaborés (de type développement de soi). Roe (1956) envisageait les effets différentiels du milieu familial sur les besoins de l'enfant (et donc aussi les futurs intérêts de l'adolescent et de l'adulte) comme des modifications de la hiérarchie des besoins de Maslow.

6 Pourtant, du fait de leur aspect relativement stable ou durable et de leur variabilité interindividuelle, les intérêts sont surtout censés relever de l'étude de la personnalité. Les besoins ne peuvent en effet s'exprimer directement dans les réalités sociales complexes - notamment celles du monde professionnel; ils doivent être ré-exprimés sous forme de buts et de moyens. Cette reformulation nécessaire à un fonctionnement motivationnel plus élaboré est prise en charge par des structures relevant de la personnalité. Les comportements simples s'organisent en conduites complexes personnalisées, c'est-à-dire qu'outre une adaptation croissante, elles présentent cohérence interne, stabilité intra-individuelle et variabilité interindividuelle.

7 La reformulation des besoins peut être analysée en termes de valeurs, une valeur étant une conception de ce qui est personnellement ou socialement désirable (Rokeach, 1973). Au cours du développement certains objets sont préférentiellement choisis, que l'on peut organiser en classes. Dans cette autre forme de rapport au monde, l'activité - orientée vers certains objets susceptibles lorsqu'ils sont atteints de satisfaire le besoin - peut être décrite selon les classes d'objets qui la déclenchent. 
8 L'auteur princeps en ce domaine aura été Spranger (Helkama, 1999, pp. 60-73) qui a décrit six grandes manières d'organiser sa vie («Lebensformen »), c'est-à-dire aussi six valeurs fondamentales susceptibles d'orienter et de dynamiser l'existence : théoriques, économiques, esthétiques, sociales, politiques et religieuses. D’autres modèles des valeurs sont plus détaillés, comme celui de Schwartz (Hammer \& Wach, 2003). Ces valeurs peuvent être plus accessibles à la représentation quand elles sont formulées en termes de personnes : c'est la démarche de Holland $(1985,1996)$ quand il pose ses types psychologiques (réaliste, investigatif, artiste, social, entrepreneur et conventionnel) mais aussi professionnels, destinés à permettre un mode de passage du monde des personnes à celui des professions. On a pu dire (Harris-Bowlsbey, Rayman \& Bryson, 1976) que ces types permettent la traduction d'un discours psychologique sur soi («psychtalk») en un discours professionnel sur les métiers (« occtalk»). De fait, ces types de Holland constituent des descripteurs complexes de soi et du monde, en termes de valeurs et d'intérêts, mais aussi d'aptitudes, d'attitudes voire de croyances.

Pour décrire des catégories d'objets (ou de buts) personnellement ou socialement désirables on a donc utilisé des valeurs, mais pour décrire les classes d'activités au moyen desquelles ces objets peuvent être atteints, on a fait état d'intérêts.

Super (1964) distinguait quatre formulations opérationnelles des intérêts : exprimés (déclarés par la personne), manifestés (observables dans la vie habituelle), testés (mesurés à l'aide d'épreuves objectives, notamment d'attention ou de mémorisation) et inventoriés (quantifiés à l'aide de questionnaires systématiques). Ce sont essentiellement ces derniers qui ont été étudiés. Depuis 1927, date de publication de l'épreuve initiale de Strong, de très nombreux inventaires ont été proposés selon l'âge des sujets, leur niveau de formation, leur expérience professionnelle ou leur maîtrise de la langue. Les inventaires peuvent utiliser des stimulus verbaux : principalement des noms de métiers, des descriptions d'activités (qu'elles soient professionnelles ou non), mais aussi divers autres items (les noms de personnages célèbres, par exemple); ils utilisent aussi des stimulus non verbaux, comme des dessins ou des photos. Ces stimulus sont souvent présentés sur un support papier mais aussi, de plus en plus fréquemment, à l'aide d'un ordinateur (Vrignaud \& Bernaud,2005, pp. 162-172; Tétreau, 2005). Le succès de la théorie de Holland a conduit à donner une place prépondérante à ses outils et à mener des études de validation en les utilisant (Guichard \& Huteau, 2001, pp. 277-288).

11 Le fort engouement pour l'évaluation des intérêts à l'aide d'inventaires systématiques tient à divers facteurs : citons leur facilité d'utilisation, leur possible aspect ludique, les faibles contraintes psychologiques qu'ils imposent au sujet ou la possibilité qu'ils offrent au psychologue de procéder à des évaluations collectives. Le succès de ces outils tient aussi à leurs qualités métrologiques. La fidélité est habituellement élevée : la valeur de $.80 \mathrm{du}$ coefficient a de Cronbach - un standard considéré souhaitable - est facilement atteinte (Prediger, 1998, par exemple). La validité prédictive est habituellement satisfaisante, même si elle a pu être surestimée (Spokane, Meir \& Catalano, 2000). Pour une approche méthodologique plus critique, on peut voir Guichard \& Huteau (2001, pp. 59-67). 


\section{Les déterminants des intérêts} Strong (1943), il est apparu nécessaire de différencier selon les sexes l'intensité des intérêts voire, parfois, leur nature et leur structure. Larcebeau (1982) oppose les intérêts scientifiques, techniques et sportifs (où la moyenne des garçons est la plus forte) aux intérêts artistiques, pour les relations altruistes et pour les relations d'affaires (où la moyenne des filles est la plus forte). De même, Botteman, Forner et Sontag (1997) opposent des intérêts relativement plus masculins, pour la mécanique ou pour le plein air, à des intérêts relativement plus féminins, pour le service social ou pour le travail de bureau. En ce sens on a pu dégager par analyse factorielle des structures différentes selon le sexe avec un facteur de soin, spécifique aux femmes (Lapan, McGrath \& Kaplan, 1990). Pourtant la tendance générale est de conclure que les scores moyens sur les dimensions diffèrent selon les sexes, mais que les structures sont en revanche identiques. L'importance de ces différences a d'abord conduit les auteurs à construire deux formes de questionnement différentes pour les femmes et les hommes, une forme unique mesurant mal les intérêts des unes comme des autres (Larcebeau, 1981, p. 11). On estime actuellement qu'il est plus souhaitable d'élaborer une forme commune accompagnée d'étalonnages différenciés (Dupont, 1987, pp. 522-523). Paineau (2005, p. 134) note que la question devient moins visible dans la mesure où «aujourd'hui la plupart des inventaires existent sous une forme informatisée, le cahier d'items (bleu pour les garçons et rose pour les filles!) a disparu : c'est le logiciel qui détermine la forme à soumettre au consultant et se réfère à l'étalonnage correspondant ».

13 Les autres déterminants psychologiques étudiés ont été principalement les traits de personnalité d'une part, les aptitudes d'autre part. logiquement compréhensibles même s'ils sont très faibles, dépassant rarement 30 (Ackerman \& Heggestad, 1997 ; Rolfhus \& Ackerman, 1996). Les intérêts sont parfois décrits comme de simples concomitants des traits de personnalité : Larson, Rottinghaus et Borgen (2002) relèvent l'analogie de structure entre le modèle de la personnalité en cinq facteurs (ouverture, conscience, extraversion, agréabilité et névrotisme) et les six types de Holland; ils relèvent également tant la cohérence logique de ces corrélations que leur faiblesse. Les intérêts peuvent aussi être conçus comme des manifestations de l'organisation (causale) de la personnalité. Ainsi, la structuration spatiale, hexagonale ou circulaire, des types de Holland serait déterminée par l'importance de deux facteurs psychologiques indépendants. Ces facteurs seraient, selon les auteurs: soit des oppositions d'attraits "personnes/objets» et "idées/données» (Prediger \& Vansickle, 1992), soit des différences sur les dimensions "sociabilité » et « conformisme» (Rounds \& Tracey, 1993).

Les aptitudes entretiennent, elles aussi, des liens cohérents mais faibles avec les intérêts. Pour Strong (1943) l'intérêt pour une activité naît de la réussite à cette activité, et la réussite est sous l'influence de l'aptitude. Toutefois, les liens entre intérêts et aptitudes seraient faibles pour deux raisons essentielles : d'une part, une aptitude peut n'être jamais exercée par manque de stimulation et ne jamais éveiller l'intérêt correspondant; d'autre part, une réussite peut intervenir par hasard ou être déclarée $a u$ vu de normes très peu exigeantes et déclencher néanmoins l'intérêt 
correspondant. Cette dernière proposition est proche de celle de Barak (1981), qui notait que les relations entre intérêts et aptitudes sont nettement plus fortes quand on considère les aptitudes perçues par le sujet, plutôt que ses aptitudes réelles. Les relations entre intérêt et performance sont conçues à l'inverse des conceptions de Strong dans la théorie sociocognitive de Bandura (Betz, 2000) : pour qu'une personne puisse réussir à une tâche, il convient d'abord qu'elle l'ait acceptée - qu'elle s'y soit intéressée. Or, pour l'auteur, l'intérêt serait fonction du sentiment de compétence, dont les variations expliqueraient notamment les différences intersexes d'intérêts (Donnay \& Borgen, 1999). On comprend l'importance donnée au développement de ce(s) sentiment(s) dans les conceptions actuelles de la guidance.

Parmi les déterminants internes des intérêts, on a envisagé des influences génétiques. Celles-ci seraient certaines (Betsworth et al.,1994), mais peut-être pas les plus intéressantes pour le psychologue et ce sont surtout les facteurs environnementaux qui ont été évoqués et étudiés.

17 Ce sont d'abord des déterminants proches que Vrignaud et Bernaud (2005, chap.4) proposent de retenir: la famille, l'école, les expériences de loisir, les expériences professionnelles ou pré-professionnelles et les relations amicales, qu'ils résument à l'aide d'un méta-facteur unique qu'ils nomment « ressources culturelles». Le processus sous-jacent serait l'apprentissage, qu'il s'effectue directement ou par l'imitation de modèles - même si son fonctionnement est évoqué de manière plutôt floue, comme le notent Bujold et Gingras (2000) à propos de Holland. Toutefois une autre tendance apparait, dans la lignée de Super (1964), qui met l'accent sur des processus plus généraux et complexes dans la genèse des intérêts, des processus contrôlés par ce régulateur des conduites qu'est le concept de soi. Ainsi, pour Gottfredson (1981), la détermination de l'attrait d'une profession passerait par son écart avec le concept de soi essentiellement en termes de sexe et de prestige. De manière voisine, pour Huteau et Vouillot (1988), l'intérêt d'un jeune pour une voie de formation résulterait de la mesure de l'écart entre la représentation de soi et la représentation des élèves typiques des diverses voies. De même, pour Guichard et Falbierski (2002) c'est parce que les jeunes qui quittent le système éducatif sur un échec ont construit une représentation de soi comme sans compétence qu'ils ne parviendraient pas à développer des intérêts susceptibles de dynamiser un projet personnel.

À côté de ces déterminants proches, d'autres plus distants ou plus généraux, de la culture sur les intérêts ont été envisagés (Vrignaud \& Bernaud, 1994). Les différences culturelles ont été étudiées par la comparaison de groupes de personnes de pays différents (Fouad \& Dancer, 1992, par exemple) et celle de groupes d'un même pays mais différents par leur appartenance sociale ou ethnique (Lattimore \& Borgen, 1999). Généralement menées dans le cadre typologique de Holland, ces études tendent surtout à montrer quelques différences plutôt anecdotiques dans une structure globalement identique des intérêts. Ainsi, Day et Rounds (1998) notent que cette structure des intérêts varie peu entre américains d'origine caucasienne, africaine, hispanique et asiatique.

La question de l'évolution générale des intérêts scolaires et professionnels est rarement posée. On dispose de peu d'indications dans ce domaine, même dans les ouvrages récents (Vrignaud \& Bernaud, 2005) à l'inverse de l'intelligence dont l'évolution a été bien étudiée depuis l'article princeps de Flynn (1984; pour une synthèse voir Flieller, 2001). La présente étude est centrée sur deux déterminants potentiels: les 
grandes évolutions professionnelles sur un quart de siècle et l'affaiblissement attendu des représentations sexuées de soi et des activités.

- Selon Holland (1985), les stéréotypes en matière d'activités, notamment professionnelles, sont « vrais ». Il entend par là que des professions différentes requièrent des comportements différents, que ces différences sont stables et qu'elles sont perçues comme telles. En ce sens, personnes et milieux professionnels auront tendance à se choisir mutuellement : sur la possibilité de réaliser ces comportements pour les premières, sur la capacité à les réaliser pour les seconds. En conséquence, les évolutions du monde du travail lors des trois dernières décennies (qu'on pourrait rapidement caractériser : minimisation de l'emploi dans le secteur primaire, déclin du secondaire, développement des services, tertiairisation de l'ensemble des activités, développement des diverses formes de communication, complexification des tâches, institutionnalisation du temps libre et des loisirs mais aussi augmentation du chômage, précarisation croissante, etc.) devrait entraîner une modification isomorphe des intérêts.

- Cette évolution générale du monde vocationnel pourrait être également marquée par une dé-différenciation des rôles sociaux selon le genre. Selon Gottfredson (1981, voir aussi Munoz-Sastre, 1994), les préférences professionnelles visent à réaliser une représentation de soi qui s'organise au cours du développement. Seraient successivement structurants : - la taille (3-5 ans), il y a alors association entre adulte et pouvoir, - puis le genre (6-9 ans), c'està-dire une sensibilité à la sexualisation des rôles sociaux, - puis encore le statut social (9-13 ans), la prise en compte des différences sociales de prestige et, enfin - les valeurs personnelles (à partir de 14 ans) lors de la construction de l'identité. On s'attend donc ici à observer, en fin de scolarité de collège, un effet du genre dans la détermination des préférences professionnelles. Selon Hansen (1988) ces différences intersexes d'intérêts s'avéreraient stables dans leur organisation, mais en progressive diminution. L'observation ne semble toutefois pas systématique : « malgré les changements sociaux... et malgré les efforts pour éliminer les biais sexuels dans les items des inventaires d'intérêts..., les scores obtenus par les hommes et les femmes à ces inventaires continuent de mettre en relief des profils... d'intérêts différents » (Tétreau, 2005, p. 90). C'est d'ailleurs ce que l'on observait dans une étude analogue menée auprès de jeunes de classes de cinquième de collège (Forner et al., 2006).

Ces deux hypothèses ont guidé très généralement la présente recherche.

\section{Enquête}

\section{Échantillon}

Les participants de l'étude étaient 482 élèves de classes de troisième de collège : 258 filles (soit 53,5\%) et 224 garçons (soit 46,5\%) scolarisés dans des établissements publics des académies de Lille et de Nancy. Les âges des sujets variaient de 13 ans à 17 ans autour d'une moyenne de 15,04 ans avec un écart-type de 0,64 an.

\section{Instrument}

Le questionnaire QIm,4,3,2 a été élaboré dans le cadre des activités du Service de Recherche de l'I.N.E.T.O.P. par Larcebeau en 1978, qui s'appuyait sur les conceptions de Kuder (Vrignaud \& Bernaud, 2005, chap. 2 et 6). Il remplaçait alors les questionnaires 
QIG,4,3, 2 et $Q I F, 4,3,2$, en usage depuis 1964 et respectivement destinés aux garçons et aux filles (voir Larcebeau, 1981).

L'épreuve comporte 12 séries chacune constituée de 9 items ou propositions. Pour chaque série, le sujet doit choisir les trois items qui lui plaisent - relativement - le plus et leur attribuer à chacun la note « 2 ». Il doit aussi déterminer les 3 items qui lui plaisent - toujours relativement - le moins et leur attribuer la note « 0 ». Les trois derniers items, qui lui sont relativement indifférents, se voient affecter la note « 1 ». La procédure consiste donc à opérer un classement simplifié des items d'une même série en adoptant une mesure de type échelle d'intervalle. Chaque série comporte un item correspondant aux catégories d'intérêts suivants: 1) littéraires, 2) artistiques, 3) scientifiques et techniques, 4) altruistes, 5) pour le commerce (et les activités de bureau), 6) sportifs, 7) pratiques, 8) pour la nature, 9) pour les relations d'affaires (on trouvera en Annexe 1 une série d'items et leur rattachement catégoriel). Chaque catégorie d'intérêts est donc appréciée à l'aide de 12 items, les scores observables à la catégorie variant de " 0 » (intérêt minimum) à « 24 » (intérêt maximum). Toutefois, pour la catégorie des intérêts pratiques, on différencie des intérêts pratiques masculins, appréciés à l'aide de 6 items (avec un score observable à la catégorie variant de « 0 » à " 12 ») et des intérêts pratiques féminins également appréciés à l'aide de 6 items (avec un score observable variant de même de « 0 » à « $12 »)$.

\section{Procédure}

482 collégiens de classes de troisième ont été interrogés, ils étaient issus de quatre collèges de l'Académie de Lille : Collège Rouges Barres de Marcq-en-Barœul, Collège de Bapaume, Collège de Provin, Collège Flandres de La Madeleine, et de deux collèges de l'Académie de Nancy: Collège de Jarville-la-Malgrange, Collège Jean Moulin de Tromblaine. Ils ont rempli le QIm,4,3,2 dans la forme papier-crayon identique à celle utilisée en 1978. Les passations ont été collectives en classes entières ou en groupes de classe pendant une heure scolaire dans le cadre de l'activité du Conseiller d'orientation psychologue de l'établissement. Après les passations, une fiche de synthèse a été remise à chaque participant pour guider une recherche documentaire et fournir le cas échéant un document de support à un entretien individuel avec le Conseiller. Le questionnaire lui-même a été présenté, certaines formulations inhabituelles ou vieillies ont été expliquées après la lecture des consignes (conformes aux consignes initiales).

\section{Résultats}

\section{Les qualités psychométriques actuelles de l'épreuve}

\section{Sensibilité}

Les données générales figurent au tableau 1.

Les distributions des scores aux échelles pour l'ensemble de l'échantillon sont d'allure générale gaussienne, mais ne le sont pas strictement : le test $\mathrm{Z}$ de Kolmogorov-Smirnov est toujours significatif à $\mathrm{p}<.01$. Ces absences de normalité apparaissent dues à deux facteurs : le sexe des sujets, pour l'essentiel, et leur origine (rurale ou urbaine). Elles 
disparaissent quand on considère séparément les distributions des scores de ces $2 \times 2$ groupes.

On note que la variabilité des scores observés sur les échelles recouvre quasiment toujours celle des observables (de 0 à 24 , sauf de 0 à 12 pour les intérêts pratiques).

\section{Tableau 1/Table 1}

\begin{tabular}{|l|c|c|c|c|c|c|}
\hline \multicolumn{1}{|c|}{ INTÉRËTS } & MOYENNE & $\begin{array}{c}\text { ECART- } \\
\text { TYPE }\end{array}$ & ASYMÉTRIE & $\begin{array}{c}\text { APLATIS } \\
\text { SEMENT }\end{array}$ & MINIMUM & MAXTMTM \\
\hline Littéraires & 9,62 & 4,76 & 0,69 & 0,23 & 0,00 & 24,00 \\
\hline Artistiques & 12,73 & 4,57 & 0,28 & $-0,35$ & 2,00 & 24,00 \\
\hline Scientifiques & 9,82 & 5,36 & 0,38 & $-0,66$ & 0,00 & 24,00 \\
\hline Altruistes & 15,92 & 4,90 & $-0,28$ & $-0,78$ & 3,00 & 24,00 \\
\hline Commerciaux & 11,39 & 4,98 & 0,04 & $-0,78$ & 0,00 & 24,00 \\
\hline Sportifs & 15,37 & 6,55 & $-0,44$ & $-0,79$ & 0,00 & 24,00 \\
\hline Pratiques masculins & 5,75 & 2,89 & 0,14 & $-0,71$ & 0,00 & 12,00 \\
\hline Pratiques féminins & 4,76 & 2,30 & 0,24 & $-0,39$ & 0,00 & 11,00 \\
\hline Nature & 10,36 & 4,90 & 0,51 & $-0,13$ & 0,00 & 24,00 \\
\hline Relations & 12,25 & 3,80 & $-0,07$ & $-0,52$ & 3,00 & 21,00 \\
\hline
\end{tabular}

Description des variables

Characteristics of variables

\section{Fidélité}

L'homogénéité des échelles a été estimée à l'aide du coefficient 頤 de Cronbach (voir tableau 2). Cet indice varie de .46 à .87, autour d'une valeur médiane .67 pour les garçons; il varie de .51 à .88 autour d'une valeur médiane de .77 pour les filles. Pour l'ensemble des participants il varie de .51 à .90 avec une médiane de .76 . Ces valeurs, obtenues sur des échelles relativement courtes ( 12 items), voire particulièrement courtes (6 items), sont acceptables pour comparer des groupes.

Toutefois, des analyses complémentaires ont été réalisées sur les échelles présentant une faible fidélité. Il apparaît que l'hypothèse d'unidimensionnalité peut être maintenue à l'exception, peut-être, des échelles d'intérêts pratiques masculins et pratiques féminins :

- Une analyse factorielle exploratoire a été pratiquée sur les 6 items de l'échelle d'intérêts pratiques masculins; après la rotation Varimax, elle indique deux facteurs : le premier, qui explique $26,77 \%$ de la variance, caractérise les items 2,3,5 et 6 et l'on peut l'interpréter comme un facteur décrivant plutôt les activités de construction. Le second facteur $(24,68 \%$ de la variance) sature les items 1 et 4 (et faiblement l'item 6) et l'on peut l'interpréter comme un facteur décrivant plutôt les activités de réparation.

- Une analyse identique a été pratiquée sur les 6 items de l'échelle d'intérêts pratiques féminins ; elle est plus satisfaisante pour l'hypothèse d'unidimensionnalité : le premier facteur, qui explique $36,07 \%$ de la variance, caractérise les 5 premiers items et l'on peut l'interpréter comme un facteur décrivant plutôt les activités d'apprentissage exploratoire. Le second facteur (20,56 \% de la variance) sature l'item 6 (et faiblement l'item 2); difficile à interpréter, il semble spécifique des activités d'entretien des vêtements.

On ne dispose pas d'indications sur l'homogénéité initiale des échelles. 
Tableau 2/Table 2

\begin{tabular}{|l|c|c|c|}
\hline \multicolumn{1}{|c|}{ INTĖRËTS } & GARÇONS (N =224) & FLLES (N =258) & ENSEMRLE (N = 482) \\
\hline Littéraires & .67 & .80 & .75 \\
\hline Artistiques & .55 & .70 & .72 \\
\hline Scientifiques & .76 & .75 & .81 \\
\hline Altruistes & .67 & .80 & .80 \\
\hline Commerciaux & .73 & .79 & .77 \\
\hline Sportifs & .87 & .88 & .90 \\
\hline Pratiques masculins & .46 & .51 & .51 \\
\hline Pratiques féminins & .56 & .55 & .63 \\
\hline Nature & .75 & .77 & .76 \\
\hline Relations d'affaires & .56 & .61 & .59 \\
\hline
\end{tabular}

Homogénéité des échelles (2004). Coefficients alpha

Scales fidelity (2004). Coefficients alpha

\section{Validité}

\section{Validité structurale : description résumée des relations entre les items}

Une analyse factorielle exploratoire a été pratiquée sur les items avec 10 facteurs censés correspondre aux $8+2$ dimensions qui ont guidé la construction du questionnaire. Après la rotation Varimax, les 10 facteurs expliquent ensemble $40 \%$ de la variance totale des items (les saturations figurent en Annexe 2) :

- le premier facteur, qui explique $7,57 \%$ de la variance totale des items, oppose 12 items sportifs, 1 scientifique et 1 pratique féminin à 1 item artistique et 1 autre commercial ; il peut être interprété comme un facteur d'intérêts sportifs ;

- le second facteur (5,40\% de la variance totale), qui oppose 11 items altruistes, 1 pratique masculin et 1 relationnel à 2 items scientifiques, 1 pratique féminin et un relationnel, peut être interprété comme un facteur d'intérêts altruistes ;

- le troisième facteur (4,42\%) oppose 10 items commerciaux à 3 items littéraires et peut être interprété comme un facteur d'intérêts commerciaux ;

- le quatrième $(4,17 \%)$ caractérise 9 items scientifiques et 1 pratique féminin et peut être interprété comme un facteur d'intérêts scientifiques ;

- le cinquième $(4,12 \%)$ oppose 10 items d'intérêts pour la nature et 2 items scientifiques à 1 item commercial et peut être interprété comme un facteur d'intérêts pour la nature ;

- le sixième facteur (3,95\%) oppose 7 items artistiques à 2 items scientifiques et 1 relationnel et peut être interprété comme un facteur d'intérêts artistiques ;

- le septième (3,61\%) oppose 7 items littéraires, 1 item artistique et 1 scientifique à 2 items relationnels et peut être interprété comme un facteur d'intérêts littéraires ;

- le huitième (3,53\%) oppose les 6 items pratiques féminins, 1 item pratique masculin et 1 scientifique à deux items littéraires et peut être interprété comme un facteur d'intérêts pratiques féminins ;

- le neuvième (3,00 \%) oppose 3 items pour la nature et 3 items pratiques ( 2 masculins et 1 féminin) à 1 item pour les relations et peut être interprété comme un facteur d'intérêts pour des activités impliquant peu de contacts humains ;

- le dernier (2,72\%) oppose 2 items pratiques masculins, 1 item altruiste et 1 item artistique à 1 item relationnel et 1 autre commercial et peut être interprété comme un facteur d'intérêt pour des activités sans contacts par rapport aux contacts intéressés. est un peu faible au vu du nombre des items (108) du questionnaire et, d'autre part, que 
l'utilisation d'analyses exploratoires sur des données ipsatives a pu être critiquée (voir, par exemple, Cheung, 2004). Si l'on accepte la méthode, on note que l'association entre dimensions du questionnaire et facteurs issus de l'analyse est globalement bonne : les dimensions sont des résumés justifiés des relations entre les items. Toutefois, les échelles d'intérêts pratiques masculins et d'intérêts pour les relations d'affaires ne présentent pas (ou plus) de validité structurale suffisante : les résultats les concernant devront être considérés avec prudence.

\section{Validité structurale : les relations entre échelles}

On ne dispose pas de données sur la structure factorielle du questionnaire lors de la passation de 1978. En revanche, Larcebeau (1981) a fourni des données sur la forme expérimentale du QIm,4,3,2. Cette forme diffère de la forme finale pour la formulation de certains items. Ainsi l'item 1, appréciant un intérêt littéraire, «Préparer un voyage à l'étranger en vous documentant sur les sites remarquables, l'histoire, les richesses artistiques du pays que vous allez visiter " était initialement "Organiser un voyage touristique. Établir un circuit tenant compte des principales curiosités et des richesses artistiques de la région que vous souhaitez visiter ».

Des comparaisons entre les structures de la forme expérimentale (étude parue en 1981) et de la passation de 2004 sont donc impossibles au niveau des items. On peut choisir de procéder à une comparaison, au niveau des échelles, même si elle n'est pas justifiée stricto sensu.

Les coefficients de corrélations figurent au tableau 3. Les structures observées en 1981 et en 2004 semblent proches, alors même qu'elles ont été obtenues à l'aide d'items parfois différents.

Puis l'auteure a effectué, séparément pour les garçons et pour les filles, une analyse factorielle des matrices des corrélations entre échelles en extrayant 4 facteurs avec la rotation Varimax. La même démarche a été appliquée aux données de 2004. Les résultats figurent aux tableaux 4-1 (garçons) et 4-2 (filles).

Pour les garçons, en 1981, les quatre facteurs expliquent $62 \%$ de la variance:- le premier oppose les intérêts altruistes, commerciaux et pour les relations d'affaire aux intérêts scientifiques (et pour la nature); on peut l'interpréter comme un facteur de contacts. - Le second facteur oppose les intérêts pratiques (M et F) aux intérêts littéraires et pour les relations d'affaires; on peut l'interpréter comme un facteur pratique. - Le troisième caractérise les intérêts sportifs et peut être interprété comme tel. - Le dernier facteur oppose les intérêts altruistes et pour la nature aux intérêts artistiques ; il est difficile à interpréter. 
Tableau 3/Table 3

\begin{tabular}{|c|c|c|c|c|c|c|c|c|c|c|}
\hline INTÉRÊTS & 1 & 2 & 3 & 4 & 5 & 6 & 7 & 8 & 9 & 10 \\
\hline 1. Littéraires & & $\begin{array}{l}.08 \\
.10\end{array}$ & $\begin{array}{l}.14 \\
.26\end{array}$ & $\begin{array}{l}-.19 \\
-.26 \\
\end{array}$ & $\begin{array}{l}-.20 \\
-.24 \\
\end{array}$ & $\begin{array}{l}-.27 \\
-.31 \\
\end{array}$ & $\begin{array}{l}-.32 \\
-.32 \\
\end{array}$ & $\begin{array}{l}-.28 \\
-.20 \\
\end{array}$ & $\begin{array}{l}-.16 \\
-.05 \\
\end{array}$ & $\begin{array}{l}-.05 \\
-.09 \\
\end{array}$ \\
\hline 2. Artistiques & $\begin{array}{l}.22 \\
.29\end{array}$ & & $\begin{array}{l}-.15 \\
-.14 \\
\end{array}$ & $\begin{array}{l}-.22 \\
-.20 \\
\end{array}$ & $\begin{array}{l}-.17 \\
-.14 \\
\end{array}$ & $\begin{array}{l}-.18 \\
-.26 \\
\end{array}$ & $\begin{array}{l}.10 \\
.02 \\
\end{array}$ & $\begin{array}{l}.04 \\
.10\end{array}$ & $\begin{array}{l}-.10 \\
-.09 \\
\end{array}$ & $\begin{array}{r}.03 \\
-.10 \\
\end{array}$ \\
\hline 3. Scientifiques & $\begin{array}{l}.22 \\
.14 \\
\end{array}$ & $\begin{array}{l}-.11 \\
-.11 \\
\end{array}$ & & $\begin{array}{l}-.29 \\
-.43 \\
\end{array}$ & $\begin{array}{l}-.31 \\
-.33 \\
\end{array}$ & $\begin{array}{l}-.19 \\
-.25 \\
\end{array}$ & $\begin{array}{l}.01 \\
.03\end{array}$ & $\begin{array}{l}-.17 \\
-.11 \\
\end{array}$ & $\begin{array}{l}.05 \\
.16 \\
\end{array}$ & $\begin{array}{l}-.25 \\
-.36 \\
\end{array}$ \\
\hline 4. Altruistes & $\begin{array}{l}-.35 \\
-.39 \\
\end{array}$ & $\begin{array}{l}-.23 \\
-.29 \\
\end{array}$ & $\begin{array}{r}-.40 \\
-.38 \\
\end{array}$ & & $\begin{array}{r}.10 \\
-.09 \\
\end{array}$ & $\begin{array}{r}-15 \\
.14 \\
\end{array}$ & $\begin{array}{l}-.16 \\
-.09 \\
\end{array}$ & $\begin{array}{r}.03 \\
-.01 \\
\end{array}$ & $\begin{array}{l}-06 \\
-.27 \\
\end{array}$ & $\begin{array}{l}.04 \\
.27 \\
\end{array}$ \\
\hline 5. Commerce & $\begin{array}{l}-.29 \\
-.23 \\
\end{array}$ & $\begin{array}{l}-.32 \\
-.23 \\
\end{array}$ & $\begin{array}{l}-.38 \\
-.34 \\
\end{array}$ & $\begin{array}{l}.16 \\
.23\end{array}$ & & $\begin{array}{l}-.20 \\
-.15 \\
\end{array}$ & $\begin{array}{l}-.03 \\
-.04\end{array}$ & $\begin{array}{r}.02 \\
-.02 \\
\end{array}$ & $\begin{array}{l}-.27 \\
-.27 \\
\end{array}$ & $\begin{array}{r}.09 \\
-.27 \\
\end{array}$ \\
\hline 6. Sportifs & $\begin{array}{l}-.35 \\
-.37 \\
\end{array}$ & $\begin{array}{l}-.26 \\
-.32 \\
\end{array}$ & $\begin{array}{l}-.11 \\
-.09 \\
\end{array}$ & $\begin{array}{r}-.11 \\
.05 \\
\end{array}$ & $\begin{array}{l}-.11 \\
-.26 \\
\end{array}$ & & $\begin{array}{r}.00 \\
-.04 \\
\end{array}$ & $\begin{array}{l}-.09 \\
-.15 \\
\end{array}$ & $\begin{array}{l}-.14 \\
-.25 \\
\end{array}$ & $\begin{array}{l}-.13 \\
-.02 \\
\end{array}$ \\
\hline $\begin{array}{l}\text { 7. Pratiques } \\
\text { masculins }\end{array}$ & $\begin{array}{l}-.22 \\
-.28 \\
\end{array}$ & $\begin{array}{r}.04 \\
-.16 \\
\end{array}$ & $\begin{array}{r}.03 \\
-.00 \\
\end{array}$ & $\begin{array}{l}-.07 \\
-.15 \\
\end{array}$ & $\begin{array}{r}-.09 \\
.05 \\
\end{array}$ & $\begin{array}{r}-.06 \\
.03 \\
\end{array}$ & & $\begin{array}{l}.23 \\
.31\end{array}$ & $\begin{array}{l}-.06 \\
-.01 \\
\end{array}$ & $\begin{array}{l}-.23 \\
-.35 \\
\end{array}$ \\
\hline $\begin{array}{l}\text { 8. Pratiques } \\
\text { féminins }\end{array}$ & $\begin{array}{l}-.28 \\
-.32 \\
\end{array}$ & $\begin{array}{r}.00 \\
-.05 \\
\end{array}$ & $\begin{array}{l}-.20 \\
-.23 \\
\end{array}$ & $\begin{array}{l}.19 \\
.08 \\
\end{array}$ & $\begin{array}{l}.08 \\
.16 \\
\end{array}$ & $\begin{array}{l}-.16 \\
-.16 \\
\end{array}$ & $\begin{array}{l}.20 \\
.30 \\
\end{array}$ & & $\begin{array}{l}.10 \\
.05 \\
\end{array}$ & $\begin{array}{l}-.22 \\
-.34 \\
\end{array}$ \\
\hline 9. Pour la nature & $\begin{array}{l}-.09 \\
-.14 \\
\end{array}$ & $\begin{array}{r}-.03 \\
.05 \\
\end{array}$ & $\begin{array}{l}.07 \\
.17 \\
\end{array}$ & $\begin{array}{l}-.04 \\
-.19 \\
\end{array}$ & $\begin{array}{l}-.25 \\
-.41 \\
\end{array}$ & $\begin{array}{l}-.24 \\
-.18 \\
\end{array}$ & $\begin{array}{l}.07 \\
.04\end{array}$ & $\begin{array}{l}.11 \\
.12\end{array}$ & & $\begin{array}{l}-.32 \\
-.37 \\
\end{array}$ \\
\hline 10. Pour les relations & $\begin{array}{l}.08 \\
.07\end{array}$ & $\begin{array}{l}-.02 \\
-.13 \\
\end{array}$ & $\begin{array}{l}-.26 \\
-.27 \\
\end{array}$ & $\begin{array}{l}.02 \\
.06\end{array}$ & $\begin{array}{l}.12 \\
.19 \\
\end{array}$ & $\begin{array}{l}-.11 \\
-.08 \\
\end{array}$ & $\begin{array}{l}-.23 \\
-.26 \\
\end{array}$ & $\begin{array}{l}-.28 \\
-.23 \\
\end{array}$ & $\begin{array}{l}-.33 \\
-.39 \\
\end{array}$ & \\
\hline
\end{tabular}

Corrélations entre échelles (Garçons/Filles)

Correlations between scales (Males/Females) oppose les intérêts altruistes et pour les relations d'affaires aux intérêts scientifiques et pour la nature ; on peut l'interpréter comme un facteur de contacts. - Le second oppose les intérêts pratiques aux intérêts littéraires et pour les relations; il peut être interprété comme un facteur pratique. - Le troisième oppose les intérêts littéraires et artistiques aux intérêts sportifs; on peut l'interpréter diversement: intérieur/ extérieur ou féminin/masculin, par exemple.-Le dernier caractérise les intérêts commerciaux et pour les relations et peut être interprété comme un facteur commercial.

Les structures observées en 1981 et en 2004 sont différentes, mais elles semblent compatibles. De plus, les parts de variance sont comparables (celle de 2004 apparaît supérieure) et les facteurs au moins aussi compréhensibles psychologiquement. Il en est de même pour les données qui concernent les filles. 
Tableau 4-1/Table 4-1

\begin{tabular}{|c|c|c|c|c|c|c|c|c|}
\hline \multirow{2}{*}{ INTÉRÊTS } & \multicolumn{2}{|c|}{$\mathrm{F} 1$} & \multicolumn{2}{|c|}{ F2 } & \multicolumn{2}{|c|}{ F3 } & \multicolumn{2}{|c|}{ F4 } \\
\hline & 1981 & 2004 & 1981 & 2004 & 1981 & 2004 & 1981 & 2004 \\
\hline Littéraires & -.31 & -.30 & -.60 & -.56 & -.39 & +.47 & & \\
\hline Artistiques & & & & & & +.83 & -.77 & \\
\hline Scientifiques & -.71 & -.77 & & & & & & \\
\hline Altruistes & +.55 & +.69 & & & & -.25 & +.48 & \\
\hline Commerce & +.67 & & & & & & & +.93 \\
\hline Sportifs & +.34 & +.37 & & & +.97 & -.63 & & -.35 \\
\hline Pratiques (M) & & & +.69 & +.75 & & & -.30 & \\
\hline Pratiques $(\mathrm{F})$ & & & +.72 & +.74 & & & & \\
\hline Nature & -.39 & -.60 & & & & & +.59 & \\
\hline Relations & +.47 & +.53 & -.50 & -.51 & & & -.27 & +.41 \\
\hline
\end{tabular}

Varimax rotation. Values less than .25 have been suppressed $(\mathrm{nl}=553 / \mathrm{n} 2=224)$

Analyse factorielle (1981/2004) des corrélations entre échelles d'intérêts (garçons)

Factor analysis (1981/2004) of correlations between scales of males' interests

Tableau 4-2/Table 4-2

\begin{tabular}{|c|c|c|c|c|c|c|c|c|}
\hline \multirow{2}{*}{ INTÉRÊTS } & \multicolumn{2}{|c|}{ F1 } & \multicolumn{2}{|c|}{ F2 } & \multicolumn{2}{|c|}{ F3 } & \multicolumn{2}{|c|}{ F4 } \\
\hline & 1981 & 2004 & 1981 & 2004 & 1981 & 2004 & 1981 & 2004 \\
\hline Littéraires & -.52 & & -.39 & -.47 & -.52 & -.41 & & +.56 \\
\hline Artistiques & -.40 & +.29 & +.43 & & -.53 & & -.42 & +.70 \\
\hline Scientifiques & -.62 & & & -.77 & & & +.50 & \\
\hline Altruistes & +.73 & & & +.75 & & & & -.27 \\
\hline Commerce & +.68 & -.69 & & +.31 & +.89 & +.41 & & \\
\hline Sportifs & & & & & & & & -.83 \\
\hline Pratiques (M) & & & +.69 & +.35 & & +.77 & & \\
\hline Pratiques $(\mathrm{F})$ & & & +.60 & & & +.70 & & \\
\hline Nature & -.39 & +.79 & & & -.26 & & +.72 & \\
\hline Relations & & -.66 & -.45 & & & -.36 & -.61 & \\
\hline
\end{tabular}

Analyse factorielle (1981/2004) des corrélations entre échelles d'intérêts (filles) Factor analysis

(1981/2004) of correlations between scales of females' interests

En 1981, les quatre facteurs expliquent $64 \%$ de la variance :- le premier oppose les intérêts altruistes et commerciaux aux intérêts scientifiques, littéraires, artistiques et pour la nature ; on peut l'interpréter comme un facteur de contacts. - Le second oppose les intérêts pratiques et artistiques aux intérêts littéraires et pour les relations d'affaires ; il peut être interprété comme un facteur pratique. - Le troisième oppose les intérêts commerciaux aux intérêts littéraires et artistiques; on peut l'interpréter comme un facteur commercial. - Le dernier oppose les intérêts scientifiques et pour la nature aux intérêts artistiques et pour les relations ; il est difficile à interpréter.

En 2004, les quatre facteurs extraits expliquent $68 \%$ de la variance:- le premier oppose les intérêts pour la nature aux intérêts commerciaux et pour les affaires; on peut l'interpréter comme un facteur de choix d'activités désintéressées. - Le second oppose les intérêts altruistes aux intérêts littéraires et scientifiques; il peut être interprété comme un facteur altruiste. - Le troisième oppose les intérêts pratiques un facteur pratique. - Le dernier facteur oppose les intérêts littéraires et artistiques aux intérêts sportifs ; il peut être diversement interprété : intérieur/extérieur ou féminin/ masculin, par exemple. 


\section{L'évolution générale des intérêts}

Pour les années 1978 et 2004, on dispose d'étalonnages, ainsi que des moyennes et des écarts-types des distributions brutes :

- le sens de l'évolution des intérêts dans le temps est déduit de la comparaison des moyennes des deux distributions (moy. 2004 - moy. 1978) ;

- la signification de la différence est appréciée par le test z ;

- l'importance de la différence des moyennes est estimée en pourcentage de l'écart-type de la distribution initiale (moy. 2004 - moy. 1978 / écart-type 1978) (voir tableau 5-1 pour les garçons et tableau 5-2 pour les filles).

Ces différences seront interprétées en termes d'évolution.

Les grandes tendances apparaissent les suivantes, en ne prenant en compte que les différences significatives à $\mathrm{p}<.001$.

- Tant chez les garçons que chez les filles, on note deux baisses :

- La baisse des intérêts scientifiques et techniques est importante : de l'ordre de la moitié de l'écart-type (pour les garçons comme pour les filles).

- La baisse des intérêts pour la nature est très importante : de l'ordre d'un écart-type et demi (garçons) et des trois quarts de l'écart-type (filles).

- Tant pour les garçons que pour les filles on note une hausse :

La hausse des intérêts pour les relations d'affaires est importante : de l'ordre de la moitié de l'écart-type (garçons et filles).

- Pour les garçons, il apparaît une hausse des intérêts altruistes (d'un tiers de l'écart-type) et commerciaux (d'une moitié de l'écart-type). On peut ajouter, au seuil de $\mathrm{p}<.01$, une hausse des intérêts sportifs (de l'ordre du cinquième de l'écart-type).

- Pour les filles, apparaît une hausse des intérêts artistiques (de l'ordre de la moitié de l'écarttype).

\section{Tableau 5-1/Table 5-1}

\begin{tabular}{|l|c|c|c|c|c|}
\hline \multicolumn{1}{|c|}{ INTERËTS } & $\begin{array}{c}1978 \\
\mathrm{~N}=553\end{array}$ & $\begin{array}{c}2004 \\
\mathrm{~N}=224\end{array}$ & TEST Z & SEUI & $\begin{array}{c}\text { TAILLE } \\
\text { DE L'EFFET }\end{array}$ \\
\hline Littéraires & $9,45(4,12)$ & $8,85(4,09)$ & 1,85 & N.S. & $-0,15$ \\
\hline Artistiques & $10,63(3,84)$ & $10,36(3,66)$ &, 92 & N.S. & $-0,07$ \\
\hline Scientifiques & $15,21(4,93)$ & $12,67(4,87)$ & 6,56 & $\mathrm{p}<.001$ & $-0,52$ \\
\hline Altruistes & $11,67(4,15)$ & $13,27(4,14)$ & 4,88 & $\mathrm{p}<.001$ & $+0,39$ \\
\hline Commerce & $8,55(4,77)$ & $11,03(4,69)$ & 6,64 & $\mathrm{p}<.001$ & $+0,52$ \\
\hline Sportifs & $17,24(5,77)$ & $18,47(5,28)$ & 2,86 & $\mathrm{p}<.01$ & $+0,21$ \\
\hline Pratiques M & $7,81(2,47)$ & $7,34(2,52)$ & 2,37 & $\mathrm{p}<.05$ & $-0,19$ \\
\hline Pratiques F & $3,75(2,20)$ & $4,08(2,12)$ & 1,94 & N.S. & $+0,15$ \\
\hline Nature & $17,08(4,75)$ & $10,31(4,84)$ & 9,88 & $\mathrm{p}<.001$ & $-1,43$ \\
\hline Affaires & $9,39(3,73)$ & $11,64(3,72)$ & 7,63 & $\mathrm{p}<.001$ & $+0,60$ \\
\hline
\end{tabular}

Moyennes (et écart-types) des garçons. Signification et taille de l'effet Means (and standard deviations) of males. Significancy and size of effect 
Tableau 5-2/Table 5-2

\begin{tabular}{|l|c|c|c|c|c|}
\hline \multicolumn{1}{|c|}{ INTÉRËTS } & $\begin{array}{c}1978 \\
\mathrm{~N}=586\end{array}$ & $\mathrm{~N}=258$ & TEST Z & SEUI & $\begin{array}{c}\text { TAILLE } \\
\text { DE L'EFFET }\end{array}$ \\
\hline Littéraires & $10,31(4,78)$ & $10,30(5,19)$ &, 03 & N.S & $-0,00$ \\
\hline Artistiques & $12,62(4,45)$ & $14,80(4,27)$ & 6,74 & $\mathrm{p}<.001$ & $+0,49$ \\
\hline Scientifiques & $10,13(5,36)$ & $7,35(4,47)$ & 7,82 & $\mathrm{p}<.001$ & $-0,52$ \\
\hline Altruistes & $17,39(4,66)$ & $18,22(4,31)$ & 1,06 & N.S. & $+0,18$ \\
\hline Commerce & $11,03(5,61)$ & $11,70(5,20)$ & 1,68 & N.S & $+0,11$ \\
\hline Sportifs & $12,05(6,66)$ & $12,68(6,36)$ & 1,14 & N.S. & $+0,09$ \\
\hline Pratiques M & $4,26(2,43)$ & $4,37(2,44)$ &, 22 & N.S. & $+0,05$ \\
\hline Pratiques F & $5,47(2,63)$ & $5,36(2,29)$ & 1,02 & N.S. & $-0,04$ \\
\hline Nature & $13,76(4,63)$ & $10,40(4,96)$ & 9,25 & $\mathrm{p}<.001$ & $-0,73$ \\
\hline Affaires & $10,73(4,84)$ & $12,79(3,79)$ & 6,66 & $\mathrm{p}<.001$ & $+0,43$ \\
\hline
\end{tabular}

Moyennes (et écart-types) des filles. Signification et taille de l'effet

Means (and standard deviations) for females. Significancy and size of effect

\section{L'évolution des différences intersexes}

L'importance de la différence des moyennes d'un intérêt selon les sexes a été estimée par $\mathrm{d}=\left(\right.$ moyF F⿱ $\left.{ }_{20} \mathrm{moyG}\right) / \mathrm{s}$ avec $\mathrm{s}=(\mathrm{nG} . \mathrm{sG}+\mathrm{nF} . \mathrm{sF}) /(\mathrm{nG}+\mathrm{nF})$.

On a donc rapporté, pour 1978, la différence entre les moyennes des deux groupes à une composition des écarts-types de ces deux groupes, plutôt qu'à l'un ou l'autre de leurs écarts-types, l'usage de rapporter les différences de moyennes à l'écart-type du groupe témoin (Guichard \& Huteau, 2001, p. 282) n'ayant pas de sens ici. La même procédure a été suivie pour les groupes observés en 2004.

7 Pour chaque catégorie d'intérêts, on peut comparer les estimations des écarts en 1978 et en 2004 et les interpréter en termes d'évolution de la différence (voir tableau 6-1 pour 1978 et tableau 6-2 pour 2004).

48 Ces différences augmentent pour quatre catégories: intérêts littéraires, artistiques, scientifiques et sportifs.

Ces différences diminuent pour cinq catégories : intérêts altruistes, pour le commerce, pratiques masculins, pratiques féminins et pour la nature.

50 La différence est stable pour une catégorie : intérêts pour les relations d'affaires.

Tableau 6-1/Table 6-1

\begin{tabular}{|l|c|c|c|c|}
\hline \multicolumn{1}{|c|}{ INTËRETS } & $\begin{array}{c}\text { GARCONS } \\
(\mathrm{N}=553)\end{array}$ & $\begin{array}{c}\text { FLLES } \\
(\mathrm{N}=586)\end{array}$ & $\begin{array}{c}\mathrm{S}= \\
(\mathrm{nG} . \mathrm{sG}+\mathrm{nF} . \mathrm{F}) \\
/(\mathrm{nG}+\mathrm{nF})\end{array}$ & $\begin{array}{c}\mathrm{D}= \\
(\mathrm{mF}-\mathrm{mG}) / \mathrm{S}\end{array}$ \\
\hline Littéraires & $9,45(4,12)$ & $10,31(4,78)$ & 4,46 & $+0,19$ \\
\hline Artistiques & $10,63(3,84)$ & $12,62(4,45)$ & 4,15 & $+0,48$ \\
\hline Scientifiques & $15,21(4,93)$ & $10,13(5,36)$ & 5,15 & $-0,99$ \\
\hline Altruistes & $11,67(4,15)$ & $17,39(4,66)$ & 4,41 & $+1,30$ \\
\hline Commerce & $8,55(4,77)$ & $11,03(5,61)$ & 5,20 & $+0,48$ \\
\hline Sportifs & $17,24(5,77)$ & $12,05(6,66)$ & 6,23 & $-0,83$ \\
\hline Pratiques M & $7,81(2,47)$ & $4,26(2,43)$ & 2,45 & $-1,45$ \\
\hline Pratiques F & $3,75(2,20)$ & $5,47(2,63)$ & 2,42 & $+0,71$ \\
\hline Nature & $17,08(4,75)$ & $13,76(4,63)$ & 4,69 & $-0,71$ \\
\hline Affaires & $9,39(3,73)$ & $10,73(4,84)$ & 4,30 & $+0,31$ \\
\hline
\end{tabular}

Moyennes (écart-types) des garçons et filles en 1978. Importance des différences Means (standard deviations) of males and females in 1978. Size of the differences 
Tableau 6-2/Table 6-2

\begin{tabular}{|l|c|c|c|c|}
\hline \multicolumn{1}{|c|}{ INTËRËTS } & $\begin{array}{c}\text { GARCONS } \\
(\mathrm{N}=224)\end{array}$ & $\begin{array}{c}\text { FLLES } \\
(\mathrm{N}=258)\end{array}$ & $\begin{array}{c}\mathrm{S}=(\mathrm{nG} . \mathrm{sG}+\mathrm{nF} . \mathrm{sF}) \\
/(\mathrm{nG}+\mathrm{nF})\end{array}$ & $\mathrm{D}=(\mathrm{mF}-\mathrm{mG}) / \mathrm{S}$ \\
\hline Littéraires & $8,85(4,09)$ & $10,30(5,19)$ & 4,58 & $+0,31$ \\
\hline Artistiques & $10,36(3,66)$ & $14,80(4,27)$ & 3,99 & $+1,11$ \\
\hline Scientifiques & $12,67(4,87)$ & $7,35(4,47)$ & 4,66 & $-1,14$ \\
\hline Altruistes & $13,27(4,14)$ & $18,22(4,31)$ & 4,23 & $+1,17$ \\
\hline Commerce & $11,03(4,69)$ & $11,70(5,20)$ & 4,96 & $+0,13$ \\
\hline Sportifs & $18,47(5,28)$ & $12,68(6,36)$ & 5,86 & $-0,99$ \\
\hline Pratiques M & $7,34(2,52)$ & $4,37(2,44)$ & 2,48 & $-1,20$ \\
\hline Pratiques F & $4,08(2,12)$ & $5,36(2,29)$ & 2,21 & $+0,58$ \\
\hline Nature & $10,31(4,84)$ & $10,40(4,96)$ & 4,90 & $+0,02$ \\
\hline Affaires & $11,64(3,72)$ & $12,79(3,79)$ & 3,76 & $+0,31$ \\
\hline
\end{tabular}

Moyennes (écart-types) des garçons et filles en 2004. Importance des différences Means (standard deviations) of males and females in 2004. Size of the differences

\section{Discussion}

51 L'observation d'une différence intergroupe doit être expliquée et l'explication peut procéder de deux grandes attitudes : l'une acceptant a priori la réalité de ces différences, l'autre la rejetant a priori en invoquant des biais de mesure. «On dit qu'une mesure est biaisée, dès lors qu'elle ne mesure pas, ou qu'imparfaitement, ce qu'elle est censée mesurée. On est en présence d'un biais lorsque la mesure met en évidence des différences entre des groupes de sujets et que ces différences ne peuvent être mises en relation avec la ou les variables mesurées » (Vrignaud, 2002, p. 626). Au plan pratique, les deux attitudes mènent à évoquer des processus différents pour expliquer les observations.

Les biais de construit peuvent être invoqués « lorsque l'instrument ne mesure pas la même variable latente selon les groupes ». Vrignaud (2002, p. 627) évoque un tel biais pour le construit « raisonnement mécanique » qui impliquerait plutôt un codage verbal chez les filles et plutôt un codage spatial chez les garçons et montrerait ainsi son " inconsistance ». De tels biais sont peu susceptibles d'intervenir, sauf à remettre en cause l'idée d'unité du processus de détermination des préférences entre 1978 et 2004 ou entre les filles et les garçons. Une critique analogue avait été formulée quant au manque de réalisme d'items des questionnaires d'intérêts portant sur des activités que les jeunes n'avaient jamais pratiquées, voire qu'ils ne pourront sans doute jamais pratiquer: la question se posait notamment pour des élèves de faible niveau scolaire lorsque les intérêts sont mesurés à l'aide d'intitulés professionnels prestigieux. Il convient de rappeler que l'ensemble de la démarche proposée repose sur le postulat que le questionnaire, avec plus ou moins de pertinence, mesure bien des intérêts.

\section{Les biais dus au matériel}

"À l'heure des supports multimédia ou d'Internet que peuvent encore bien signifier (item 84) "écouter à la radio une émission documentaire sur un sujet scientifique" ou encore (item 39) "Construire un poste à transistors d'après des schémas et des croquis" »? : nous a-t-on objecté. On pourrait répondre au plan logique et demander à quel autre type d'intérêt, que scientifique et technique, ces deux activités pourraient 
bien correspondre. On peut surtout répondre au plan psychométrique : la corrélation corrigée entre l'item 39 et le total de l'échelle d'intérêts scientifiques est de +.53 , cet item est un des plus liés à ce score total. Quant à l'item 84 , sa corrélation item - total corrigée est de +.20 ; elle est plus faible mais cet item n'est lié plus fortement au score total d'aucune autre catégorie du questionnaire. L'échelle elle-même est très homogène $(a l p h a=.81)$. Sauf à considérer que l'ensemble de l'échelle est à rejeter, ces items contribuent bien à évaluer les intérêts scientifiques et techniques.

Les biais d'échantillonnage sont plus importants à considérer. On peut en considérer de deux ordres. Les premiers porteraient sur les choix des échantillons lors des deux passations: on ne connaît pas, par exemple, l'origine géographique des jeunes interrogés en 1978 et il est probable qu'elle diffère de celle de 2004. On peut pourtant rappeler que cette variable n'a pas fait l'objet d'études montrant son importance dans la détermination des intérêts. On pourrait raisonner de même pour plusieurs autres variables.

Les autres biais porteraient sur les populations d'origine de 1978 et de 2004. «En 1977, 28 \% des jeunes sortaient du système éducatif sans aucun diplôme, 11 \% n'avaient obtenu que le brevet, $30 \%$ avaient un C.A.P. ou un Brevet professionnel et $11 \%$ s'arrêtaient au bac. Aujourd'hui c'est la quasi-totalité d'une génération (97\%) qui atteint le niveau de la troisième" (Marseille, 2004, p. 41). De même, Salines et Vrignaud (2001, p. 35) notent: «La composition de la population des élèves de troisième s'est trouvée progressivement modifiée entre les années 1980 et 1999 du fait, dans un premier temps, de la disparition de l'orientation en fin de cinquième puis, dans un second temps, de la disparition des troisièmes technologiques. La proportion d'une génération scolarisée en troisième générale est ainsi passée de $66 \%$ en 1984, à $76 \%$ en 1990, $86 \%$ en 1995 et enfin à $100 \%$ en 1999 ». Des jeunes qui auparavant auraient quitté le collège en fin de classe de cinquième parce qu'entre autres, ils présentaient des intérêts peu scolaires, poursuivent maintenant jusqu'en classe de troisième : le biais pourrait expliquer, au moins pour partie, la baisse observée des intérêts scientifiques, la hausse des intérêts commerciaux et, à un moindre titre, la hausse des intérêts pour les relations d'affaires. Ce biais irait toutefois à l'inverse de la baisse-qui reste l'observation la plus forte-des intérêts (également peu scolaires, tels qu'ils sont appréciés par le questionnaire) pour la nature.

On peut aussi répondre à la question des biais d'échantillonnage en évoquant une étude comparable, menée dans d'autres établissements, qui portait sur l'évolution des intérêts des élèves de cinquième de collège (Forner et al., 2006) et qui aboutissait à des résultats proches. Or, que ce soit en 1978 ou en 2004, la classe de cinquième était suivie par la quasi-totalité des élèves. Il apparaissait que certains intérêts étaient en baisse : scientifiques et pour la nature, "ceux qui nécessitent relativement moins de contacts humains et qui inscrivent l'activité dans une certaine perspective temporelle». À l'inverse, certains intérêts étaient en hausse : ceux qui «correspondent, d'une part, à une possibilité de contacts qu'ils soient plutôt altruistes (intérêts sociaux) ou plus intéressés (intérêts commerciaux) et, d'autre part, à une orientation vers les loisirs (intérêts artistiques)».

57 Les présentes observations portent sur des catégories légèrement différentes mais, mutatis mutandis, les conclusions qui en découlent sont presque identiques : - baisse des intérêts correspondant à un investissement personnel autonome inscrit dans une perspective temporelle (intérêts scientifiques et pour la nature), - hausse des intérêts 
correspondant à des possibilités de communication immédiate, lors de contacts humains plutôt intéressés (intérêts pour les relations d'affaires et, plus spécifiquement pour les garçons, intérêts commerciaux et intérêts altruistes. N. B. Pour ces deux dernières catégories on pourrait dire, en fait, que les garçons rattrapent les filles). - On peut ajouter une hausse spécifique des intérêts artistiques pour les filles, des intérêts de loisirs pour lesquels il est souvent difficile de proposer une traduction professionnelle (hormis les professions d'enseignement).

Au plan pratique, la baisse des intérêts scientifiques ne peut que conforter dans l'idée que la désaffection des sciences par les étudiants correspond à une tendance lourde puisqu'elle se manifeste aussi nettement au collège, en cinquième comme en troisième. De même, la baisse des intérêts pour la nature paraît correspondre au fait d'évidence que ce secteur n'est plus depuis longtemps porteur d'emplois en grand nombre (même s'il ne faudrait pas que cette baisse détourne trop des nouvelles activités spécifiques et qualifiées de ce domaine). Globalement, cette évolution moyenne des intérêts semble correspondre à l'évolution générale de la société française sur un quart de siècle.

En revanche, l'observation d'une forte résistance de la répartition des intérêts professionnels moyens des garçons et des filles semble conforter les conceptions de Gottfredson; elle parait décevante pour les attentes des pouvoirs publics, mais cohérente avec des observations réalisées sur des jeunes de classe de cinquième (Forner et al., 2006) où les évolutions des intérêts des filles et des garçons semblent au mieux parallèles, voire peut-être légèrement divergentes.

60 Les interventions psychopédagogiques visant à modifier les représentations professionnelles des filles et des garçons (dans le sens d'un moindre sexisme!) ont souvent pour effet des modifications modestes des représentations, généralement dans le sens espéré mais pas systématiquement (voir Guégnard,2002, par exemple). Pourtant, souvent aussi, aucune modification des projets personnels n'est observée. On peut noter ici un fait peut-être anecdotique mais peu encourageant: les différences intersexes augmentent pour les catégories qui sont les plus familières à des collégiens, celles qui correspondent le mieux aux disciplines scolaires (!) : intérêts littéraires, artistiques, scientifiques et sportifs. À l'inverse, les différences diminuent, peu, pour les activités qui semblent les plus étrangères au système éducatif : intérêts altruistes, pratiques, pour le commerce et pour la nature.

61 Les différences intersexes diminuent pour cinq catégories d'intérêts, elles augmentent pour quatre autres et sont stables pour une dernière : globalement, on ne peut conclure à un rapprochement des intérêts moyens des garçons et des filles à la fin du collège sur le dernier quart de siècle.

\section{BIBLIOGRAPHIE}

Ackerman, P. L., \& Heggestad, E. D. (1997). Intelligence, personality, and interests : Evidence for overlapping traits. Psychological Bulletin, 121, 219-245. 
Barak, A. (1981). Vocational interests : A cognitive view. Journal of Vocational Behavior, 19, 1-14.

Betsworth, D. G., Bouchard, T. J. Jr., Cooper, C. R., Grotevant, H. D., Hansen, J. C., Scarr, S., \& Weinberg, R. A. (1994). Genetic and environmental influences on vocational interests assessed using biological and adoptive families and twins reared apart and together. Journal of Vocational Behavior, 44, 263-278.

Betz, N. E. (2000). Self-efficacy theory as a basis for career assessment. Journal of Career Assessment, $8,3,205-222$.

Botteman, A., Forner, Y., \& Sontag, J.-C. (1997). Manuel du Relevé d'Intérêts Professionnels. Paris : E.C.P.A.

Bujold, C., \& Gingras, M. (2000). Choix professionnel et développement de carrière : théories et recherches ( 2 édition). Boucherville, QU : G. Morin.

C.C.S.T.I.-Grenoble (2004). Regards sur les métiers scientifiques et techniques. Rapport d'enquête. www.ccsti-grenoble.org/GDS/enqueteCCSTI-grenoble.pdf.

Cheung, M. W. L. (2004). A direct analysis method on analyzing ipsative data with Chan and Bentler's (1993) method. Structural Equation Modeling, 11-2, 217-243.

Day, S. X., \& Rounds, J. (1998). Universality of vocational interest structure among racial and ethnic minorities. American Psychologist, 53, 728-736.

Donnay, D. A. C., \& Borgen, F. H. (1999). The incremental validity of vocational self-efficay : An examination of interests, self-efficacy, and occupation. Journal of Counseling Psychology, 46, 432-447.

Dupont, J.-B. (1987). Les intérêts, leur évolution et leur mesure. In C. Lév-Leboyer \& J.-C. Spérandio, Traité de psychologie du travail et des organisations, pp. 511-533. Paris : P.U.F. Dupont, J.-B., Gendre, F., Berthoud, S., \& Descombes, J.-P. (1979). La psychologie des intérêts. Paris : P.U.F.

Flieller, A. (2001). Problèmes et stratégies dans l'explication de l'effet Flynn. In M. Huteau, Éd., Les figures de l'intelligence, pp. 43-66. Paris : E.A.P.

Flynn, J. R. (1984). The mean IQ of Americans : Massive gains. 1932 to 1978. Psychological Bulletin, 95, 29-51.

Forner, Y., Ferrer, S., Le Veler, C., \& Thiéry, S. (2006). Les intérêts professionnels des collégiens : l'évolution sur un quart de siècle. Psychologie du Travail et des Organisations, à paraître.

Fouad, N. A. \& Dancer, L. S. (1992). Cross-cultural structure of interests : Mexico and the United States. Journal of Vocational Behavior, 40, 129-143.

Gottfredson, L. S. (1981). Circumscription and compromise : A developmental theory of occupational aspirations. Journal of Counseling Psychology, 32, 1, 152-159.

Guégnard, C. (2002). Représentations professionnelles des filles et des garçons au collège. Les effets d'une pièce de théâtre interactive. L'Orientation Scolaire et Professionnelle, 31, 4, 601-622.

Guichard, J., \& Falbierski, E. (2002). Compétences et projets : mots vides ou concepts pertinents pour l'insertion des jeunes en difficulté. Carriérologie, 8, 3, 427-454.

Guichard, J., \& Huteau, M. (2001). Psychologie de l'orientation. Paris : Dunod.

Hammer, B., \& Wach, M. (2003). La structure des valeurs est-elle universelle ? Genèse et validation du modèle compréhensif de Schwartz. Paris : L'Harmattan. 
Hansen, J. C. (1988). Changing interests of women : Myth or reality. Applied Psychology : An International Review, 37, 2, 133-150.

Harris-Bowlsbey, J. A., Rayman, J. R., \& Bryson, D. L. (1976). Discover : A computer-based career guidance and counselor-administrative support system. Fiels Trial Report. Westminster, MD : Discover Foundation.

Helkama, K. (1999). Recherches récentes sur les valeurs. In J. L. Beauvois, N. Dubois, \& W. Doise (Éds.), La construction sociale de la personne. Grenoble : P.U.G.

Holland, J. L. (1985). Making vocational choices : A theory of vocational personalities and work environments, 2nd ed. Englewood Cliffs, NJ : Prentice Hall.

Holland, J. L. (1996). Exploring careers with a typology. American Psychologist, 51, 4, 397-406.

Huteau, M., \& Vouillot, F. (1988). Représentations et préférences professionnelles. Bulletin de Psychologie, 42, 144-153.

Lapan, R. T., McGrath, E., \& Kaplan, (1990). Factor structure of the basic interests scales by gender across time. Journal of Counseling Psychology, 37, 2, 216-222.

Larcebeau, S. (1967). Psychologie différentielle des intérêts : l'influence du sexe.

B.I.N.O.P., 23-3, 165-207.

Larcebeau, S. (1981). Un questionnaire d'intérêts pour garçons et filles. QIm,4,3,2. Paris : Service de Recherche de l'I.N.E.T.O.P.

Larcebeau, S. (1982). Intérêts, valeurs et choix professionnels. L'Orientation Scolaire et Professionnelle, 11, 4, 341-354.

Larson, L. M., Rottinghaus, P. J., \& Borgen, F. H. (2002). Meta-analysis of big six interests and big five personality factors. Journal of Vocational Behavior, 61, 2, 217-239.

Lattimore, R. R., \& Borgen, F. H. (1999). Validity of the 1994 Strong Interest Inventory with racial and ethnic groups in the United States. Journal of Counseling Psychology, 46, 2, 185-195.

Marseille, J. (2004). La guerre des deux France. Paris : Plon.

Munoz-Sastre, M.-T. (1994). La théorie de Gottfredson : exposé critique. L'Orientation Scolaire et Professionnelle, 23, 2, 233-251.

Ourisson, G. (2002). Désaffection des étudiants pour les études scientifiques. Rapport de Guy Ourisson. www.education.gouv.fr/rapport/ourisson/ourisson.pdf.

Paineau, A. (2005). Quelques « points sensibles » d'un inventaire d'intérêts professionnels. Carriérologie, 10, 1, 131-152.

Prediger, D. J. (1982). Dimensions underlying Holland's hexagon : Beyond RIASEC. Journal of Vocational Behavior, 21, 259-287.

Prediger, D. J. (1998). Is interest profile relevant to career counseling ? Journal of Counseling Psychology, 45, 204-211.

Prediger, D. J., \& Vansickle, T. R. (1992). Locating occupations on Holland's hexagon : Beyond RIASEC. Journal of Vocational Behavior, 40, 111-128.

Roe, A. (1956). The psychology of occupations. New York : Wiley.

Rokeach, M. (1973). The nature of human values. New York : Free Press.

Rolfhus, E. L., \& Ackerman, P. L. (1996). Self-report knowledge : At the crossroads of ability, interest, and personality. Journal of Educational Psychology, 88, 174-188. 
Rounds, J. B., \& Tracey, T. J. G. (1993). Prediger's dimensional representation of Holland's RIASEC circumplex. Journal of Applied Psychology, 78, 875-890.

Salines, M., \& Vrignaud, P. (2001). Apprécier et certifier les acquis des élèves en fin de collège : diplôme et évaluations-bilans. Les rapports établis à la demande du Haut conseil de l'évaluation de l'école, 2.

Spokane, E. L., Meir, E. I., \& Catalano, M. (2000). Person-environment congruence and Holland's theory : A review and reconsideration. Journal of Vocational Behavior, 57, 2, 137-187.

Strong, E. K. Jr (1943). Vocational interests of men and women. Stanford, CA : Stanford University Press.

Super, D. E. (1964). La psychologie des intérêts. Paris : P.U.F.

Tétreau, B. (2005). L'essor d'une psychologie des intérêts professionnels. Carriérologie, 1, 1, 75-118.

Vrignaud, P. (2002). Les biais de mesure : savoir les identifier pour y remédier. Bulletin de Psychologie, 56, 6, 625-634.

Vrignaud, P., \& Bernaud, J. L. (1994). La structure des intérêts dans une perspective transculturelle : le modèle de Holland est-il transférable en France? Psychologie et Psychométrie, $n^{\circ}$ spécial, 11-39.

Vrignaud, P., \& Bernaud, J. L. (2005). L'évaluation des intérêts professionnels. Sprimont : Mardaga.

\section{RÉSUMÉS}

Un questionnaire d'intérêts, le QIm,4,3,2, a été rempli par 482 élèves de classes de troisième en 2004. Les résultats ont été comparés à ceux obtenus en 1978. Il apparaît, d'une part, une baisse des intérêts scientifiques et pour la nature et, d'autre part, une hausse des intérêts pour les relations d'affaires. Quatre catégories d'intérêts paraissent évoluer différemment selon le genre: les intérêts pour le commerce, les intérêts altruistes et les intérêts sportifs augmentent seulement chez les garçons (alors qu'ils sont stables chez les filles); à l'inverse, les intérêts artistiques n'augmentent que chez les filles. Ces variations sont cohérentes avec celles qui ont été observées sur des collégiens plus jeunes, elles semblent correspondre à l'évolution sociale générale, mais modifient peu les différences intersexes.

An interest inventory, the QIm,4,3,2, has been completed by 482 students of ninth grade in 2004 . Results have been compared with those obtained by students in 1978. It discloses a fall in scientific and nature interests and a rise in business interests. Variations differ according to gender in four categories: male students display higher interests than previously in trade, altruism and sports. Female students' interests appear to be stable in these domains but on the rise in the artistic one (where no change is observed in male students). These variations are consistent with those observed in younger students. They are probably a consequence of a more general social trend. Nevertheless, gender differences in interests remain almost stable.

\section{INDEX}

Keywords : Assessment, Gender, Interests, Sex differences, Vocational guidance

Mots-clés : Différences intersexes, Évaluation, Genre, Intérêts, Orientation professionnelle 


\section{AUTEURS}

\section{YANN FORNER}

est professeur de psychologie à l'université Lille 3. Courriel : yann.forner@univ-lille3.fr AGNÈS LASNE-CORDONNIER

est conseillère d'orientation-psychologue au CIO de Maubeuge

\section{CHANTAL-MARIE MERCIER}

est conseillère d'orientation-psychologue au CIO de Sarrebourg

\section{HAMIDA SALH}

est conseillère d'orientation-psychologue au CIO de Molsheim 